\title{
Platelet-derived microparticles promote phagocytosis of oxidized low-density lipoprotein by macrophages, potentially enhancing foam cell formation
}

\author{
Can Feng ${ }^{1,2 \#}$, Qi Chen ${ }^{3 \#}$, Min Fan ${ }^{2 \#}$, Jun Guo ${ }^{1}$, Yu Liu ${ }^{4}$, Tao Ji ${ }^{5}$, Jiaqi Zhu ${ }^{1}$, Xianxian Zhao ${ }^{1}$ \\ ${ }^{1}$ Department of Cardiology, Changhai Hospital, Second Military Medical University, Shanghai 200433, China; ${ }^{2}$ Department of Cardiology, Yueyang \\ Hospital of Integrated Traditional Chinese and Western Medicine, Shanghai University of Traditional Chinese Medicine, Shanghai 200437, \\ China; ${ }^{3}$ Department of Cardiology, Biomedical Research Center, Sir Run Run Shaw Hospital, School of Medicine, Zhejiang University, Hangzhou \\ 310016, China; ${ }^{4}$ Department of Cardiology, Nanjing University Medical School Affiliated Nanjing Drum Tower Hospital, Nanjing 210008 , China; \\ ${ }^{5}$ Department of Neurosurgery, Tenth Affiliated Hospital, Tongji University, Shanghai 200072, China \\ Contributions: (I) Conception and design: C Feng, Q Chen, J Zhu, X Zhao; (II) Administrative support: J Zhu, X Zhao; (III) Provision of study \\ materials or patients: C Feng, Q Chen, M Fan; (IV) Collection and assembly of data: J Guo, Y Liu, T Ji; (V) Data analysis and interpretation: C \\ Feng, Q Chen; (VI) Manuscript writing: All authors; (VII) Final approval of manuscript: All authors. \\ "These authors contributed equally to this work. \\ Correspondence to: Dr. Jiaqi Zhu; Xianxian Zhao. Department of Cardiology, Changhai Hospital, Second Military Medical University, Shanghai \\ 200433, China. Email: zjq77324@126.com; xianxianz2010@163.com.
}

\begin{abstract}
Background: The interaction between platelets and macrophages plays an important role in the development and progression of atherosclerosis (AS). This study aimed to investigate the role of platelet microparticles (PMPs) in the development of foam cells.

Methods: PMPs are generated by activating platelets with thrombin and separated by ultracentrifugation. The macrophages were treated with PMPs, the phagocytosis of oxidized low-density lipoprotein (Ox-LDL) and formation of foam cells were evaluated by flow cytometry and confocal microscopy, respectively, and the inflammatory factors cytokines in the supernatant were detected by ELISA.

Results: PMPs significantly increase the phagocytosis of Ox-LDL and elevated foam cell formation of macrophages. IL-1 $\beta$ content in the supernatant of macrophages peaked around $2-4 \mathrm{~h}$ and declined to normal level after $6-8 \mathrm{~h}$; IL-6 content peaked at $4 \mathrm{~h}$ and then decreased to normal level. TNF- $\alpha$ content peaked at 2-4 h.

Conclusions: The microparticles from activated platelets can increase the phagocytosis of Ox-LDL and the production of inflammatory cytokines by macrophages, which is related to the development of AS.
\end{abstract}

Keywords: Platelets; microparticles; macrophages; coronary atherosclerosis disease

Submitted Apr 06, 2019. Accepted for publication Jul 24, 2019.

doi: 10.21037/atm.2019.08.06

View this article at: http://dx.doi.org/10.21037/atm.2019.08.06

\section{Introduction}

Cardiovascular disease (CVD) has become the leading cause of death world wide and coronary heart disease (CHD) is a major form of CVD. The pathogenesis of CHD is the complex, and the pharmacologic approaches and change of lifestyle can lower plasma cholesterol rather than treat CHD. It has been proposed that CHD is an inflammatory disease caused by the interaction among coronary arterial cells, extracellular matrix, blood, hemodynamics, environmental factors and genetic factors. In addition, smoking, hypertension, hyperglycemia, low density lipoproteinemia, oxidative stress and inflammatory factors are also found to be the risk factors of CHD and can accelerate the occurrence and development of CHD. To 
date, most of studies have focused on the endothelial cell injury, lipoprotein deposition, macrophage phagocytosis and foam cell accumulation in the pathogenesis of atherosclerosis (AS), a major cause of CHD (1). In 1976, Moore et al. (2) found rabbits with the platelet count lower than $1,000 \mathrm{~mm}^{3}$ basically avoided the aortic endothelial injury. This suggests platelets play an important role in the pathogenesis of AS after endothelial injury.

The formation of atherosclerotic plaques is closely related to the accumulation of oxidized low-density lipoprotein (Ox-LDL), activation of macrophages, $\mathrm{T}$ cell infiltration, cell death and fibrosis of arterial wall. These processes may result in the accumulation of ApoB lipoproteins in the arterial wall. These lipoproteins can be phagocytized by macrophages, eventually causing the formation of foam cells, which is a crucial process in the atherosclerotic plaque formation. Moreover, the constant stimulation by the ApoB can also result in local chronic inflammation.

Recent study has revealed that microparticles from platelets have a close relationship with the platelet activation and AS $(3,4)$, but the interaction of platelet microparticles (PMPs) with macrophages has not been fully studied so far although there is evidence showing the important role of PMPs in the recruitment of leukocytes (5). The mechanism underlying the platelet production is complex, halflife of platelets is relatively short and the culture system for platelet has not been established. This study aimed to investigate the role of PMPs in the development of foam cells.

\section{Methods}

\section{Isolation of PMPs}

Blood samples were collected from healthy volunteers in the Changhai Hospital of the Second Military Medical University and platelets were separated. The PMPs were separated according to previously reported (6). In brief, platelets were centrifuged at $150 \mathrm{~g}$ for $15 \mathrm{~min}$ at $15^{\circ} \mathrm{C}$ to remove the residual red blood cells in platelets. The supernatant was collected and then centrifuged at $1,000 \mathrm{~g}$ for $10 \mathrm{~min}$ at $22{ }^{\circ} \mathrm{C}$. The sediment was re-suspended in the serum-free RPMI1640 (GE health care life science, Pittsburgh, PA, USA), followed by addition of $0.1 \mathrm{U} / \mathrm{mL}$ thrombin (Sigma Aldrich, St. Louis, MO, USA). Incubation was done at $37^{\circ} \mathrm{C}$ for 15 min under constant shock to facilitate platelet aggregation. After centrifugation at 3,200 g for $10 \mathrm{~min}$ at $22{ }^{\circ} \mathrm{C}$, the supernatant was collected and then ultracentrifuged at $20,000 \mathrm{~g}$ for $90 \mathrm{~min}$ at $22{ }^{\circ} \mathrm{C}$. The sediment was PMPs which were collected and re-suspended in the serum-free RPMI1640. This study was approved by the Ethics Committee of Changhai Hospital (No: CHEC2014-066). Written informed consent was obtained from each subject.

\section{Collection of macrophages}

The macrophages were harvested after induced differentiation of THP-1 cells (ATCC, Manassas, VA, USA). THP-1 cells were maintained in the complete RPMI-1640 supplemented with $10 \%$ fetal bovine serum (FBS) at $37^{\circ} \mathrm{C}$ in an environment with $5 \% \mathrm{CO}_{2}$ (7). THP-1 cells were treated with $100 \mathrm{nM}$ phorbol 12-myristate 13 -acetate (PMA, Sigma Aldrich, St. Louis, MO, USA) for $24 \mathrm{~h}$ to induce the differentiation into macrophages (1). When the cell confluence reached $80-90 \%$, the medium was removed and refreshed with treatment medium.

\section{Laser confocal microscopy}

To investigate the interaction between PMPs and macrophages, $1 \mu \mathrm{L}$ of CM-Dil dye was added to $100 \mu \mathrm{L}$ of PMPs at a final concentration of $10^{6} / \mathrm{mL}$ (PMPs) and then the mixture was incubated at $37^{\circ} \mathrm{C}$ for $20 \mathrm{~min}$. Thereafter, the mixture was ultracentrifuged at $20,000 \mathrm{~g}$ for $90 \mathrm{~min}$ at $18{ }^{\circ} \mathrm{C}$. The supernatant was removed, and $100 \mu \mathrm{L}$ of serum-free RPMI 1640 was used to re-suspend the PMPs. PMPs were incubated with macrophages at $37{ }^{\circ} \mathrm{C}$ for $24 \mathrm{~h}$, followed by washing with PBS twice. The macrophages were observed by confocal microscopy (Leica Microsystems, German). One hundred $\mu \mathrm{L}$ of supernatant without PMPs served as the control group. All experiments were performed in triplicate and repeated at least three times.

\section{Flow cytometry and confocal microscopy}

The macrophages were divided into two groups. In the PMPs group, macrophages were incubated with $100 \mu \mathrm{L}$ of PMPs $\left(10^{6} / \mathrm{mL}\right)$ for $48 \mathrm{~h}$. In the control group, $100 \mu \mathrm{L}$ of supernatant without PMPs was added. In both groups, macrophages were treated with $20 \mu \mathrm{L}$ of DiI-Ox-LDL (40 $\mu \mathrm{g} / \mathrm{mL}$, Guangzhou Yiyuan biotech company). After incubation with DAPI (Sigma Aldrich, St. Louis, MO, USA) for $6 \mathrm{~h}$, cells were observed by laser confocal microscopy. 
The mean fluorescence intensity of DiI was determined by flow cytometry.

\section{ELISA}

The macrophages were divided into two groups. In the PMPs group, macrophages were treated with $100 \mu \mathrm{L}$ of PMPs $\left(10^{6} / \mathrm{mL}\right)$. In the control group, macrophages were treated with $100 \mu \mathrm{L}$ of supernatant without PMPs. At 0, 2, 4,6 , and $8 \mathrm{~h}$, the contents of IL- $1 \beta$, IL- 6 , and TNF- $\alpha$ in the supernatant were detected by ELISA (IMMULITE ${ }^{\circledR}$ 1000 Systems, Siemens Healthcare, USA). All the tests were repeated three times.

\section{Statistical analysis}

SPSS version 22 was used for statistical analysis (SPSS Inc., Chicago, IL, USA). Data are expressed as mean \pm standard error (SEM). The mean fluorescence intensity was subjected to Mann-Whitney test. A value of $\mathrm{P}<0.05$ was considered statistically significant. GraphPad Prism 6.0 was used to draft the figures (GraphPad Software, Inc., California, USA).

\section{Results}

\section{Collection and identification of PMPs}

PE-conjugated CD41a served as a marker of platelets, and the presence of CD41a is indicative of platelet-derived microparticles. FITC-conjugated Annexin V served as a marker for microvesicles, which can differentiate microvesicles from cell debris. The flow cytometry was done to observe the characteristics of the dual channel fluorescent signal. As shown in Figure 1, about $50 \%$ of products was isolated from the platelet-derived microbubbles, and the remaining components (about 40\%) were platelet-derived fragments.

\section{Phagocytosis of PMPs by macrophages}

After treatment with PMA, the proliferation ability of THP-1 cells was weakened, the suspended cells became adherent, the cell body was enlarged, and some of cells showed pseudopodia (Figure 2A). These suggest that THP-1 cells differentiate into macrophages. To investigate the phagocytosis of PMPs by macrophages, macrophages were treated with PMPs. Then, the PMP and nucleus were stained with CM-Dil and DAPI, respectively, and cells were subsequently observed by confocal microscopy. The red fluorescence was found besides the nucleus of macrophages, suggesting that PMPs can be phagocytized by macrophages (Figure 2B,C,D).

\section{PMPs enhance phagocytosis of $O x-L D L$ by macrophages}

As shown in Figure 3A, more Dil-Ox-LDL was observed in the PMPs group than in control group, and the histogram of mean fluorescence intensity showed right shift (Figure 3B), which indicates more Dil-Ox-LDL in the PMPs group (Figure 3C). It is indicated that PMPs enhance the phagocytosis of $\mathrm{Ox}$-LDL by macrophages.

\section{PMPs increase release of inflammatory factors by macrophages}

After PMPs treatment, the content of IL-1 $\beta$ in the supernatant of macrophages peaked around $2-4 \mathrm{~h}$ and began to drop to normal level after $6-8 \mathrm{~h}$, indicating that PMPs increase the secretion of IL- $1 \beta$ by macrophages (Figure $4 A$ ). Meanwhile, the content of IL- 6 peaked at $4 \mathrm{~h}$ and then decreased to normal level (Figure $4 B$ ); the content of TNF- $\alpha$ peaked at $2-4 \mathrm{~h}$ in the supernatant of macrophages (Figure $4 C$ ). These results indicate the inflammatory cytokines released by macrophages increase after incubation with PMPs.

\section{Discussion}

Our findings indicated that PMPs played an important role in the formation of foam cells and the release of inflammatory cytokines by macrophages. After pretreatment with PMPs, the phagocytotic capability of macrophages and the release of IL- $1 \beta$, IL- 6 and TNF- $\alpha$ increased significantly. In the present study, the platelets were activated with thrombin to mimic the in vivo physiological process during the development of CHD. PMPs can bridge the interaction between platelets and macrophages, and our results also confirm the pro-inflammatory effects of PMPs.

PMPs have previously been considered as the "waste" of platelets (8). In recent years, increasing findings suggest PMPs have physiological and biochemical activities in the blood (9). The role of PMPs in the blood coagulation has been widely studied $(10,11)$. PMPs are released and produced by the platelets after activation, antagonism, or 
A

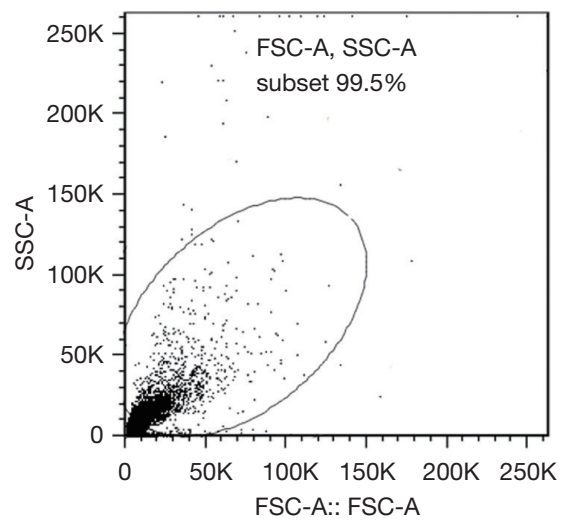

B



C

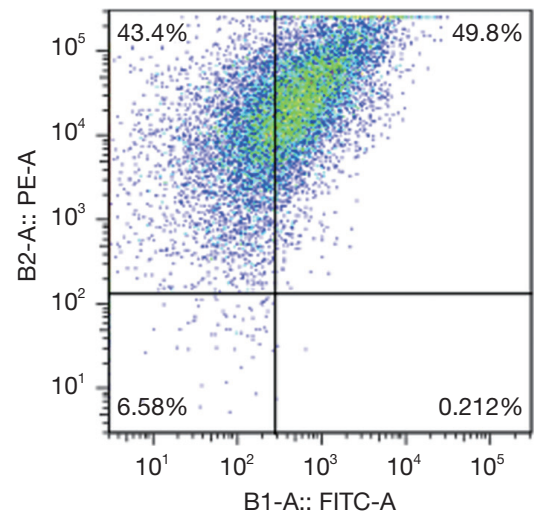

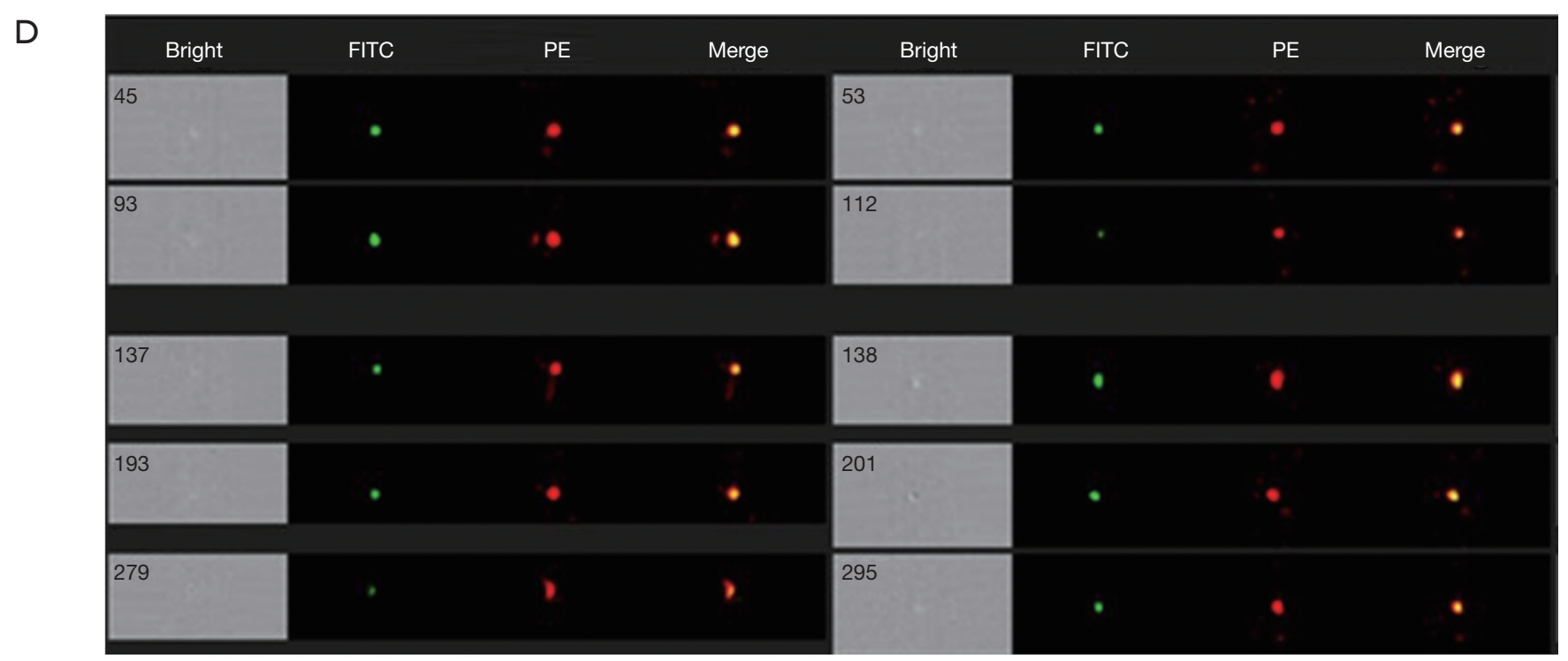

Figure 1 Flow cytometry of PMPs. The platelets were separated and activated with thrombin. Then, the supernatant was collected and ultracentrifuged for the collection of PMPs. PMPs were observed by flow cytometry. (A) number of PMP on flow cytometry (FSC: forward scattered light; SSC: lateral scattered light); (B) PMP distribution on flow cytometry; (C) proportion of PMP and cell debris; (D) multidimensional panoramic flow imaging of PMPs (Bright: light microscope; FITC: Annexin V channel; PE: CD41a channel). PMP, platelet microparticle.

high shear. Growth factors (e.g., platelet-derived growth factor, transforming growth factor $\beta$ ) (12), enzymes (e.g., 12-lipoxygenase) (13), cytokines (e.g., IL-1) $(14,15)$, transcription factors (13) and even functional mitochondria $(13,16)$ are found in the PMPs, and PMPs can be efficiently internalized by other cells such as endothelial cells $(17,18)$ and neutrophils (13). Available studies have shown that patients with severe peripheral vascular AS tend to have higher levels of PMPs in the circulation (19). PMPs can bind to the membrane structure in the endothelial cells to activate these cells, which then recruit platelets into the injured endothelium (20). In addition, PMPs may also induce the expression of intercellular adhesion molecule 1 (ICAM1) and increase the expression of pro-inflammatory cytokines IL-1, IL-6 and IL-8 $(21,22)$ which are consistent with our results. Barry et al. (21) showed PMPs enhanced the adhesion of monocytes to endothelial cells, which might be associated with the arachidonic acid in the PMPs. Xue et al. (23) found PMPs were involved in the immune regulation and systemic inflammation of rheumatoid 


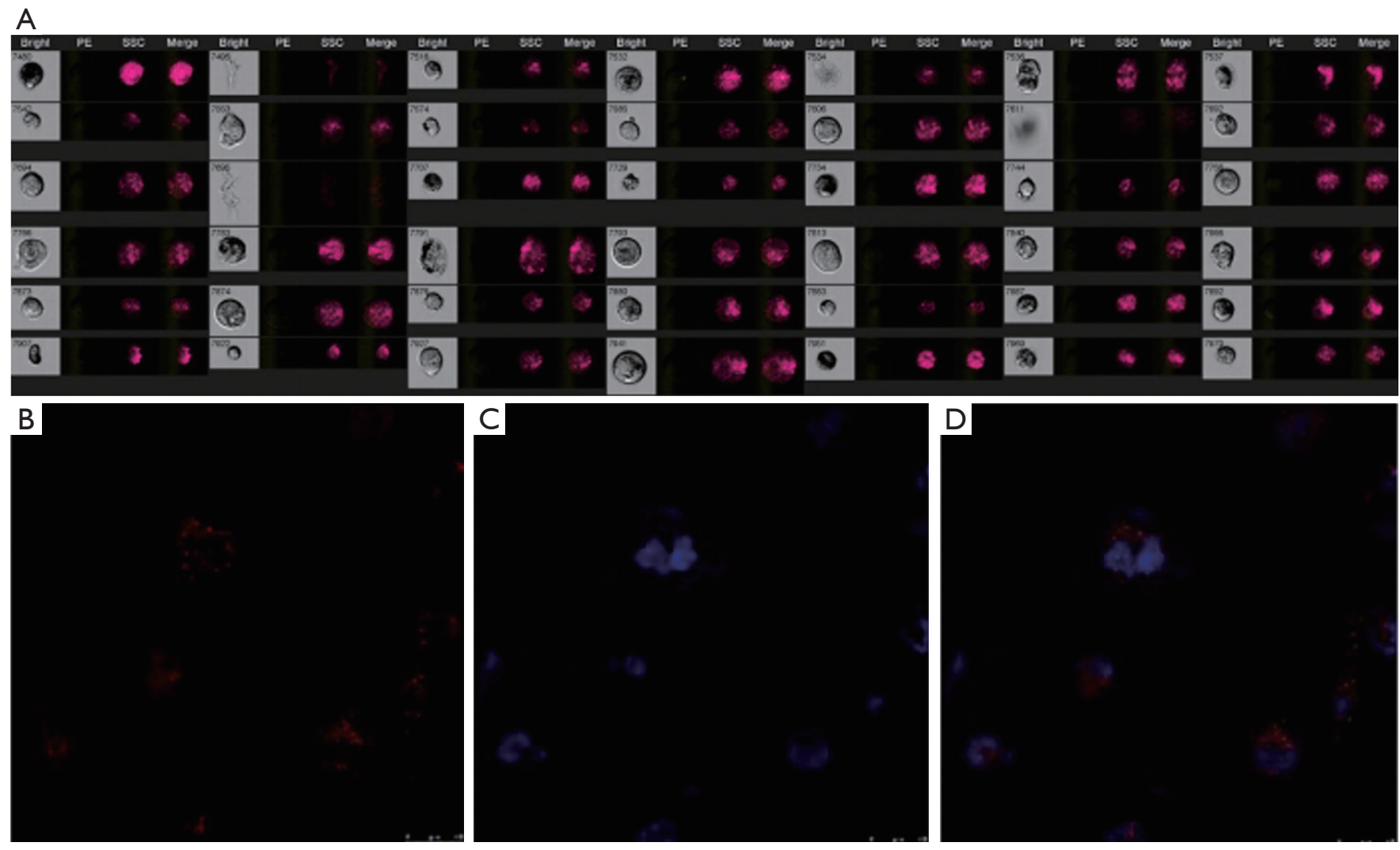

Figure 2 Flow cytometry of THP-1 cells and laser confocal microscopy of PMPs. (A) THP-1 cells were treated with PMA for 24 h and then observed by flow cytometry. THP-1 cells differentiate into macrophages and PMPs can be phagocytized by macrophages. Laser confocal microscopy of PMPs (B; CM-Dil) and nucleus (C; DAPI); (D) merged. PMP, platelet microparticle.

arthritis (RA), and the increase in PMPs could serve as a potential biomarker for RA. However, PMPs have never been thoroughly explored as a biologically active molecule.

Our results were consistent with previously reported $(24,25)$ that PMPs can be phagocytized by macrophages. Besides, our findings expanded the work of Laffont et al. (24). Our results showed PMPs treatment increased the phagocytosis of Ox-LDL and the release of inflammatory cytokines by macrophages. Ox-LDL used in the present study is an important risk factor of coronary AS. Ox-LDL can promote the endothelial injury, formation of foam cells, proliferation of vascular smooth muscle, and occurrence and development of AS. Mononuclear macrophages can transform into foam cells after phagocytosis of Ox-LDL. Foam cells are characteristic pathology of AS, and foam cells in the process of AS are mainly derived from mononuclear macrophages. In vitro experiments have shown that Ox-LDL can induce the miRNA-155 expression in mouse peritoneal macrophages (26), and miRNA-155 then regulates HMG- domain protein 1 (HBP1) expression. Thus, the increased phagocytosis of Ox-LDL by macrophages after PMPs treatment may be related to a variety of biologically active molecules (such as miRNA-155) in the PMPs (27) because these molecules may enhance the synthesis of phagocytosisrelated proteins, thereby enhancing the phagocytosis of Ox-LDL.

In recent years, increasing studies focus on the interaction between microparticles and cells. In this study, the interaction between PMPs and macrophages was investigated under the background of coronary AS. Our results showed PMPs not only enhanced the phagocytotic capability of macrophages, but also increased the release of inflammatory cytokines by macrophages. However, the specific mechanism should be further studied in depth.

In summary, our findings indicate the important role of PMPs in the pathogenesis of CHD. Although platelets are the anucleated elements of the blood, they play a central role in the hemostasis and thrombosis, and possess other 
A
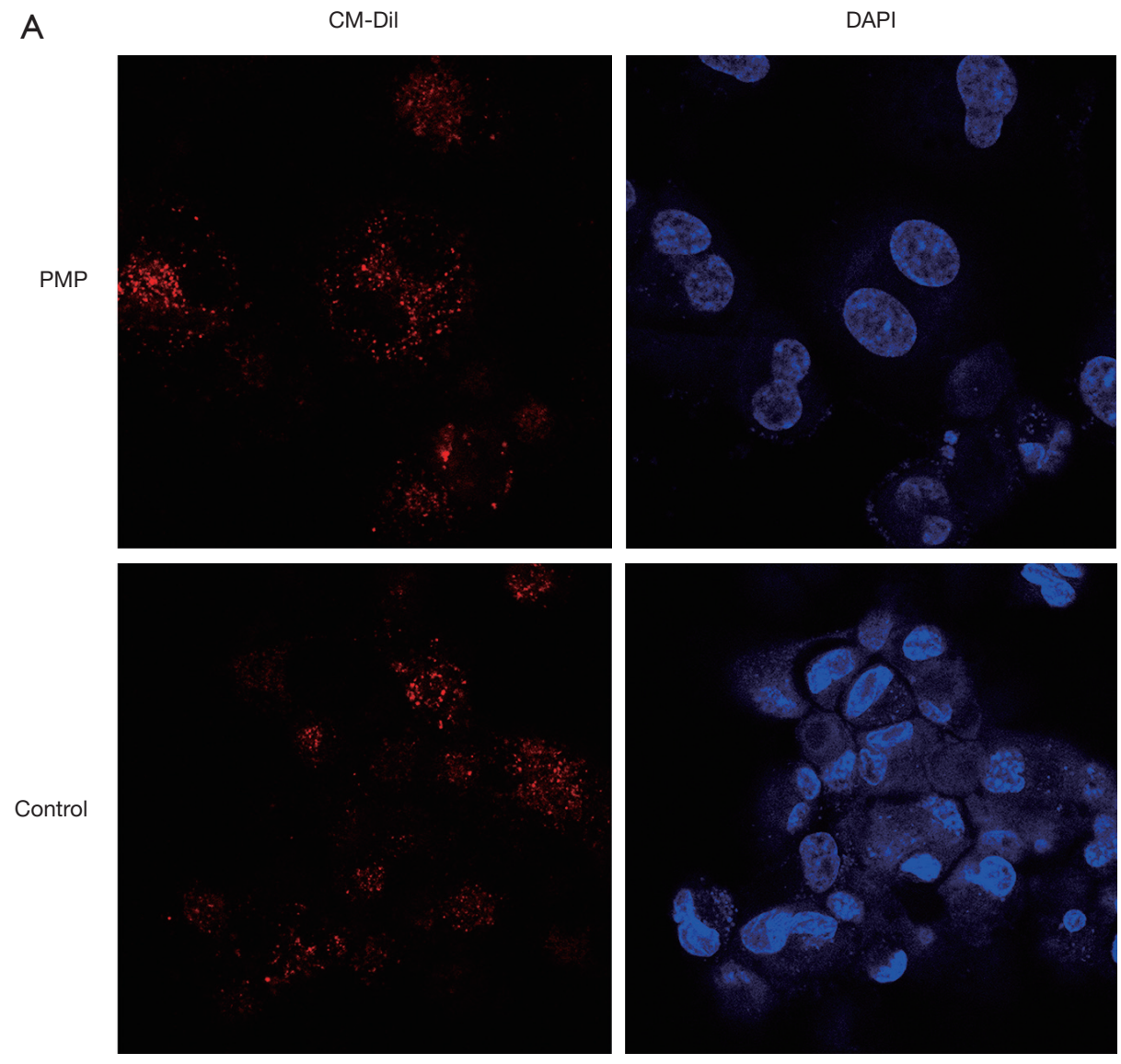

B

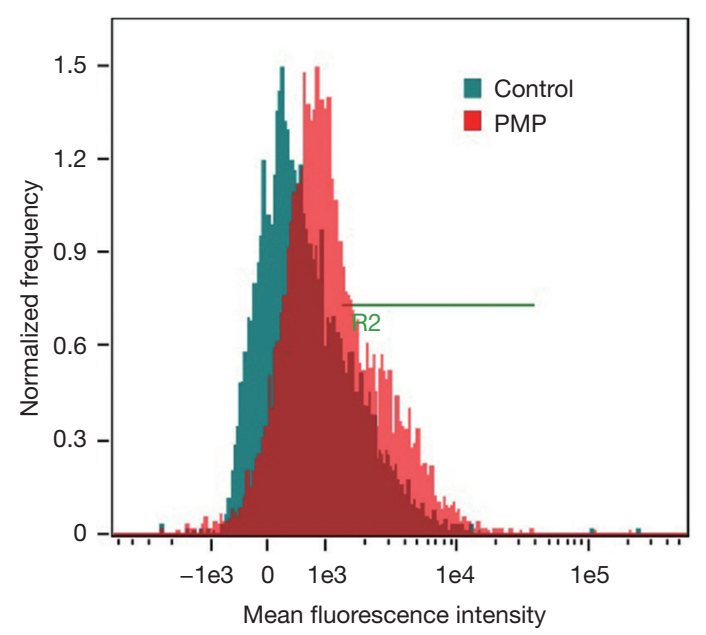

C

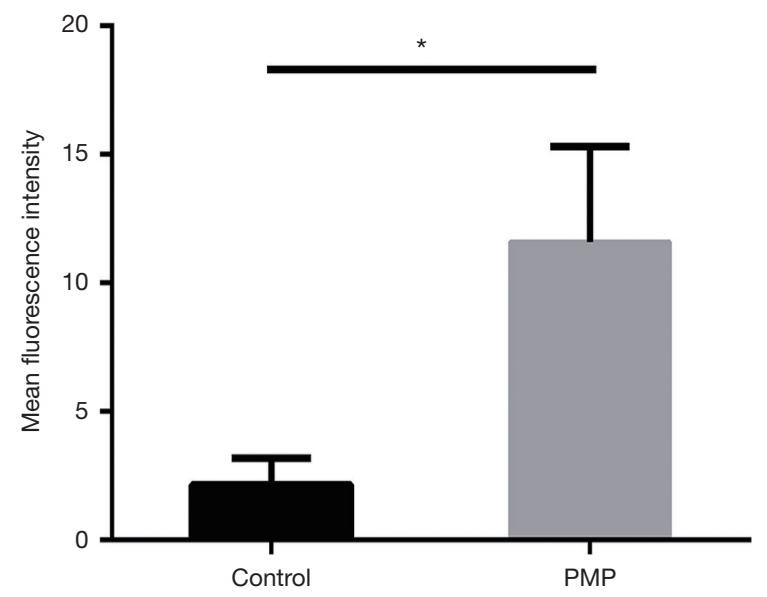

Figure 3 PMPs enhance phagocytosis of Ox-LDL by macrophages. The macrophages were incubated with PMPs for $48 \mathrm{~h}$ and then with DiI-Ox-LDL. The mean fluorescence intensity of DiI was determined by flow cytometry. (A) Laser confocal microscopy of macrophages; (B) flow cytometry of macrophages; (C) mean fluorescence intensity. *, $\mathrm{P}<0.05$. PMP, platelet microparticle. 
A
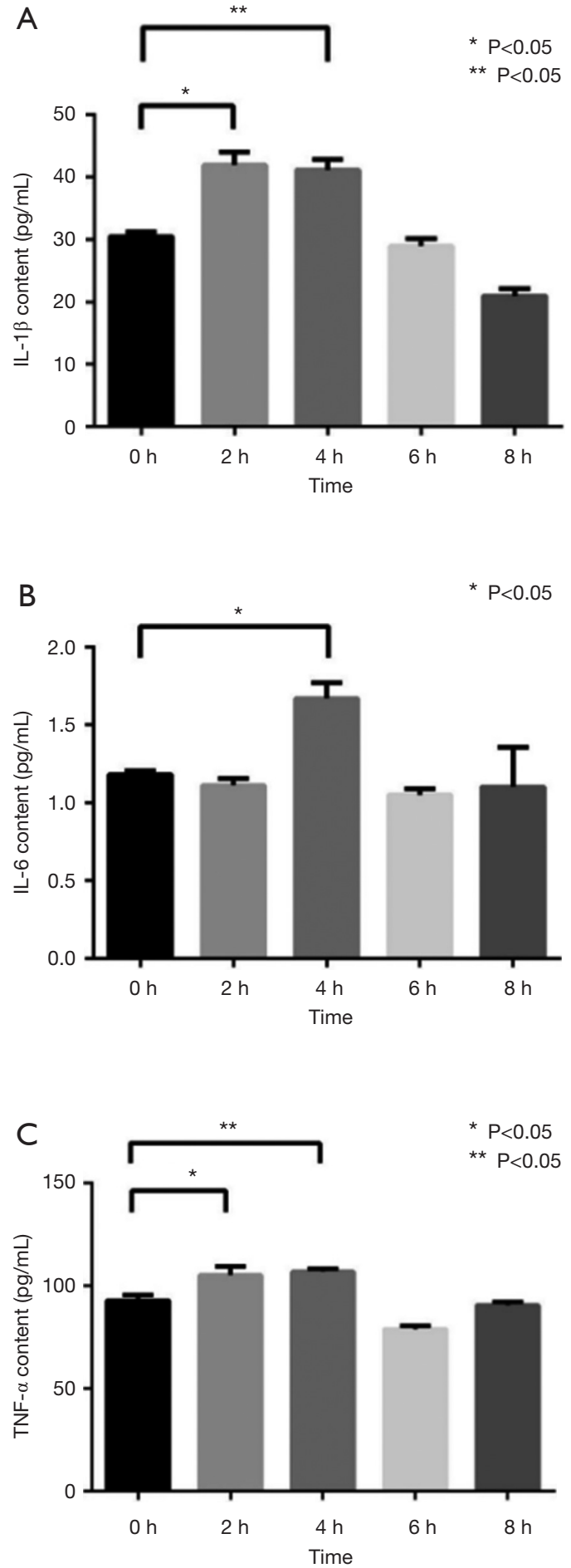

Figure 4 Contents of inflammatory cytokines released by macrophages. (A) PMPs increased the secretion of IL- $1 \beta$ by macrophages; (B) the content of IL- 6 peaked at $4 \mathrm{~h}$ and then decreased to normal level; (C) the content of TNF- $\alpha$ peaked at $2-4 \mathrm{~h}$ in the supernatant of macrophages. ${ }^{*}, \mathrm{P}<0.05 ;{ }^{*}, \mathrm{P}<0.05$. PMP, platelet microparticle. important biological functions. Thus, the inhibition of adverse effects of PMPs may serve as a strategy in the future treatment of CHD.

There are still limitations in our study. The PMPs might be contaminated by platelets during the separation because ultracentrifugation can't completely remove platelets. In addition, the specific mechanism underlying the interaction between PMPs and macrophages was not explored. Third, the dose-response relationship of PMPs in the treatment of macrophages was not investigated, and PMPs were collected from healthy controls, but not from patients with CHD.

\section{Acknowledgments}

Funding: The study was supported by the Natural Science Foundation of Shanghai (No.: 15ZR1413600), National Natural Science Foundation of China (No. 81904007, No. 81570208 and No. 81000117), and Yueyang Hospital Research Projects (2018YJ05).

\section{Footnote}

Conflicts of Interest: The authors have no conflicts of interest to declare.

Ethical Statement: The authors are accountable for all aspects of the work in ensuring that questions related to the accuracy or integrity of any part of the work are appropriately investigated and resolved. This study was approved by the Ethics Committee of Changhai Hospital (No: CHEC2014-066). Written informed consent was obtained from each subject.

\section{References}

1. Goldschmidt-Clermont PJ, Creager MA, Losordo DW, et al. Atherosclerosis 2005: recent discoveries and novel hypotheses. Circulation 2005;112:3348-53.

2. Moore S, Friedman RJ, Singal DP, et al. Inhibition of injury induced thromboatherosclerotic lesions by antiplatelet serum in rabbits. Thromb Haemost 1976;35:70-81.

3. Randriamboavonjy V, Fleming I. Platelet communication with the vascular wall: role of platelet-derived microparticles and non-coding RNAs. Clin Sci (Lond) 2018;132:1875-88.

4. Wang ZT, Wang Z, Hu YW. Possible roles of plateletderived microparticles in atherosclerosis. Atherosclerosis 
2016;248:10-6.

5. Morel O, Morel N, Freyssinet JM, et al. Platelet microparticles and vascular cells interactions: a checkpoint between the haemostatic and thrombotic responses. Platelets 2008;19:9-23.

6. Kailashiya J. Platelet-derived microparticles analysis: Techniques, challenges and recommendations. Anal Biochem 2018;546:78-85.

7. Fuhrman B, Partoush A, Volkova N, et al. Ox-LDL induces monocyte-to-macrophage differentiation in vivo: Possible role for the macrophage colony stimulating factor receptor (M-CSF-R). Atherosclerosis 2008;196:598-607.

8. Pawlowski CL, Li W, Sun M, et al. Platelet microparticleinspired clot-responsive nanomedicine for targeted fibrinolysis. Biomaterials 2017;128:94-108.

9. El-Gamal H, Parray AS, Mir FA, et al. Circulating microparticles as biomarkers of stroke: A focus on the value of endothelial- and platelet-derived microparticles. J Cell Physiol 2019;234:16739-54.

10. Lin HC, Chang HW, Hsiao SH, et al. Platelet-derived microparticles trigger THP-1 monocytic cell aggregation and release of pro-coagulant tissue factor-expressing microparticles in vitro. Transfus Apher Sci 2015;53:246-52.

11. Lukasik ZM, Makowski M, Makowska JS. From blood coagulation to innate and adaptive immunity: the role of platelets in the physiology and pathology of autoimmune disorders. Rheumatol Int 2018;38:959-74.

12. Pierce GF, Mustoe TA, Lingelbach J, et al. Plateletderived growth factor and transforming growth factor-beta enhance tissue repair activities by unique mechanisms. J Cell Biol 1989;109:429-40.

13. Duchez AC, Boudreau LH, Naika GS, et al. Platelet microparticles are internalized in neutrophils via the concerted activity of 12-lipoxygenase and secreted phospholipase A2-IIA. Proc Natl Acad Sci U S A 2015;112:E3564-73.

14. Brown GT, McIntyre TM. Lipopolysaccharide signaling without a nucleus: kinase cascades stimulate platelet shedding of proinflammatory IL- $1 \beta$-rich microparticles. J Immunol 2011;186:5489-96.

15. Boilard E, Nigrovic PA, Larabee K, et al. Platelets amplify inflammation in arthritis via collagen-dependent microparticle production. Science 2010;327:580-3.

16. Boudreau LH, Duchez AC, Cloutier N, et al. Platelets release mitochondria serving as substrate for bactericidal group IIA-secreted phospholipase A2 to promote inflammation. Blood 2014;124:2173-83.
17. Dasgupta SK, Le A, Chavakis T, et al. Developmental Endothelial Locus-1 (Del-1) Mediates Clearance of Platelet Microparticles by the EndotheliumClinical Perspective. Circulation 2012;125:1664-72.

18. Laffont B, Corduan A, Plé H, et al. Activated platelets can deliver mRNA regulatory Ago2 $\bullet$ microRNA complexes to endothelial cells via microparticles. Blood 2013;122:253-61.

19. Tan KT, Tayebjee MH, Lynd C, et al. Platelet microparticles and soluble $\mathrm{P}$ selectin in peripheral artery disease: relationship to extent of disease and platelet activation markers. Ann Med 2005;37:61-6.

20. Merten M, Pakala R, Thiagarajan P, et al. Platelet microparticles promote platelet interaction with subendothelial matrix in a glycoprotein IIb/IIIa-dependent mechanism. Circulation 1999;99:2577-82.

21. Barry OP, Pratico D, Savani RC, et al. Modulation of monocyte-endothelial cell interactions by platelet microparticles. J Clin Invest 1998;102:136-44.

22. Miao D, Ma TT, Chen M, et al. Platelets release proinflammatory microparticles in anti-neutrophil cytoplasmic antibody-associated vasculitis. Rheumatology (Oxford) 2019. [Epub ahead of print].

23. Xue LJ, Cui BB, Li X, et al. Association of Elevated Platelet Microparticles with Disease Activity in Rheumatoid Arthritis. Sichuan Da Xue Xue Bao Yi Xue Ban 2017;48:405-9.

24. Laffont B, Corduan A, Rousseau M, et al. Platelet microparticles reprogram macrophage gene expression and function. Thromb Haemost 2016;115:311-23.

25. Dasgupta SK, Abdel-Monem H, Niravath P, et al. Lactadherin and clearance of platelet-derived microvesicles. Blood 2009;113:1332-9.

26. Tian FJ, An LN, Wang GK, et al. Elevated microRNA-155 promotes foam cell formation by targeting HBP1 in atherogenesis. Cardiovasc Res 2014;103:100-10.

27. Gao J, Ma X, Zhang Y, et al. The role of microRNAs in prethrombotic status associated with coronary artery disease. Thromb Haemost 2017;117:429-36.

Cite this article as: Feng C, Chen Q, Fan M, Guo J, Liu Y, Ji T, Zhu J, Zhao X. Platelet-derived microparticles promote phagocytosis of oxidized low-density lipoprotein by macrophages, potentially enhancing foam cell formation. Ann Transl Med 2019;7(18):477. doi: 10.21037/atm.2019.08.06 